Article

\title{
Implicative Neutrosophic Quadruple BCK-Algebras and Ideals
}

\author{
G. Muhiuddin 1,*化, Ahmad N. Al-Kenani ${ }^{2} \mathbb{D}$, Eun Hwan Roh ${ }^{3}$ and Young Bae Jun 4 \\ 1 Department of Mathematics, University of Tabuk, Tabuk 71491, Saudi Arabia \\ 2 Department of Mathematics, King Abdulaziz University, P.O. Box 80219, Jeddah 21589, Saudi Arabia; \\ aalkenani10@hotmail.com \\ 3 Department of Mathematics Education, Chinju National University of Education, Jinju 52673, Korea; \\ ehroh9988@gmail.com \\ 4 Department of Mathematics Education, Gyeongsang National University, Jinju 52828, Korea; \\ skywine@gmail.com \\ * Correspondence: chishtygm@gmail.com
}

Received: 15 November 2018; Accepted: 13 February 2019; Published: 21 February 2019

\begin{abstract}
A neutrosophic set is initiated by Smarandache, and it is a novel tool to deal with vagueness considering the truth, indeterminacy and falsity memberships satisfying the condition that their sum is less than 3. The concept of neutrosophic quadruple numbers was introduced by Florentin Smarandache. Using this idea, Jun et al. introduced the notion of neutrosophic quadruple $B C K / B C I$-numbers, and studied neutrosophic quadruple $B C K / B C I$-algebras. As a continuation of Jun et al.'s paper, the notion of implicative neutrosophic quadruple $B C K$-algebras is introduced, and several properties are investigated. Given a set $Y$, conditions for the neutrosophic quadruple $Y$-set $\mathcal{N}_{q}(Y)$ to be a neutrosophic quadruple BCI-algebra are provided. Conditions for the neutrosophic quadruple $Y$-set $\mathcal{N}_{q}(Y)$ to be an implicative neutrosophic quadruple $B C K$-algebra are provided. Given subsets $I$ and $J$ of a $B C K$-algebra $Y$, conditions for the neutrosophic quadruple $(I, J)$-set $\mathcal{N}_{q}(I, J)$ to be an implicative ideal of the neutrosophic quadruple $B C K$-algebra $\mathcal{N}_{q}(Y)$ are discussed.
\end{abstract}

Keywords: (commutative, implicative) neutrosophic quadruple BCK-algebra; (commutative, implicative) neutrosophic quadruple ideal; neutrosophic quadruple $(I, J)$-set

\section{Introduction}

$B C K / B C I$-algebras are an algebraic structure, which was introduced by Imai, Iséki and Tanaka in 1966, that describes fragments of the propositional calculus involving implication known as $B C K$ and $B C I$ logics. The notion of neutrosophic set, which is developed by Smarandache (see [1-3]), is a more general platform which extends the notions of (intuitionistic) fuzzy set, interval valued (intuitionistic) fuzzy set and classic set. Neutrosophic set theory has useful applications in several branches. Decision-making problems are some of the most widely used phenomena in our real-life applications or in various fields like science, engineering, operation research, and management sciences. Garg and Nancy [4] developed a nonlinear programming model based on the technique for order preference by similarity to ideal solution (TOPSIS), in order to solve decision-making problems in which criterion values and their importance are given in the form of interval neutrosophic numbers (INNs). Garg and Nancy [5] presented some new operational laws called logarithm operational laws with real number base for the single-valued neutrosophic (SVN) numbers, and applied it to multiattribute decision making. In algebraic structures of $B C K / B C I$-algebras and semigroup, neutrosophic set theory is discussed in the papers [6-15]. Smarandache [16] introduced the notion of neutrosophic quadruple numbers. Akinleye et al. [17] introduced the concept of neutrosophic quadruple algebraic structures. 
Jun et al. [18] studied the neutrosophic quadruple algebraic structures in $B C K / B C I$-algebras, and they introduced the notion of neutrosophic quadruple $B C K / B C I$-algebras.

In this article, we introduce the concept of implicative neutrosophic quadruple $B C K$-algebras, and investigate several properties. In the first and second sections, introduction and basic notions/results are displayed. In the third section, we discuss several properties and (implicative) neutrosophic quadruple ideals in (implicative) neutrosophic quadruple $B C K$-algebras. Given a set $Y$, we discuss conditions for the neutrosophic quadruple $Y$-set $\mathcal{N}_{q}(Y)$ to be a neutrosophic quadruple $B C I$-algebra. We provide conditions for the neutrosophic quadruple $Y$-set $\mathcal{N}_{q}(Y)$ to be an implicative neutrosophic quadruple $B C K$-algebra. Given subsets $I$ and $J$ of a $B C K$-algebra $Y$, we find conditions for the neutrosophic quadruple $(I, J)$-set $\mathcal{N}_{q}(I, J)$ to be an implicative ideal of the neutrosophic quadruple $B C K$-algebra $\mathcal{N}_{q}(Y)$.

\section{Preliminaries}

A BCI-algebra (see [19]) is defined to be a set $Y$ with a binary operation $*$ and a special element 0 which satisfies the following conditions:

(I) $\quad(\forall a, b, c \in Y)(((a * b) *(a * c)) *(c * b)=0)$,

(II) $(\forall a \in Y)(a * 0=a)$,

(III) $(\forall u, v \in Y)(u * v=0, v * u=0 \Rightarrow u=v)$.

If a $B C I$-algebra $Y$ satisfies the following identity:

(IV) $(\forall a \in Y)(0 * a=0)$,

then $Y$ is called a $B C K$-algebra. Any $B C K / B C I$-algebra $Y$ satisfies the following conditions:

$$
\begin{aligned}
& a * 0=a, \\
& a \leq b \Rightarrow a * c \leq b * c, c * b \leq c * a, \\
& (a * b) * c=(a * c) * b, \\
& (a * c) *(b * c) \leq a * b
\end{aligned}
$$

for all $a, b, c \in Y$.

A $B C K$-algebra $Y$ is said to be

- commutative if the following assertion is valid:

$$
(\forall a, b \in Y)(a *(a * b)=b *(b * a)) .
$$

- implicative if the following assertion is valid:

$$
(\forall a, b \in Y)(a *(b * a)=a) .
$$

A subset $I$ of a $B C K$-algebra $Y$ is called

- $\quad$ an ideal of $Y$ if it satisfies:

$$
\begin{aligned}
& 0 \in I, \\
& (\forall a \in Y)(\forall b \in I)(a * b \in I \Rightarrow a \in I),
\end{aligned}
$$

- a commutative ideal of $Y$ if it satisfies Label (7) and

$$
(\forall a, b \in Y)(\forall c \in I)((a * b) * c \in I \Rightarrow a *(b *(b * a)) \in I) .
$$


- $\quad$ an implicative ideal (see [20]) of $Y$ if it satisfies (7) and

$$
(\forall a, b, c \in Y)(((a *(b * a)) * c \in I, c \in I \Rightarrow a \in I) .
$$

We refer the reader to the books $[19,20]$ for further information regarding $B C K / B C I$-algebras, and to $[2,3]$ for further information regarding neutrosophic set theory.

Definition 1 ([18]). Let $Y$ be a nonempty set. A neutrosophic quadruple $Y$-number is an ordered quadruple $(a, x T, y I, z F)$ where $a, x, y, z \in Y$ and $T, I, F$ have their usual neutrosophic logic meanings.

The set of all neutrosophic quadruple $Y$-numbers is denoted by $\mathcal{N}_{q}(Y)$, that is,

$$
\mathcal{N}_{q}(Y):=\{(a, x T, y I, z F) \mid a, x, y, z \in Y\},
$$

and it is called the neutrosophic quadruple set based on $Y$ or neutrosophic quadruple $Y$-set.

Let $Y$ be a set with a binary operation $*$ and a special number 0 . We define a binary operation $\tilde{*}$ on $\mathcal{N}_{q}(Y)$ by

$$
(a, x T, y I, z F) \tilde{*}(b, u T, v I, w F)=(a * b,(x * u) T,(y * v) I,(z * w) F)
$$

for all $(a, x T, y I, z F),(b, u T, v I, w F) \in \mathcal{N}_{q}(Y)$. Given $x_{1}, x_{2}, x_{3}, x_{4} \in Y$, the neutrosophic quadruple $Y$-number $\left(x_{1}, x_{2} T, x_{3} I, x_{4} F\right)$ is denoted by $\tilde{x}$, that is,

$$
\tilde{x}=\left(x_{1}, x_{2} T, x_{3} I, x_{4} F\right),
$$

and the zero neutrosophic quadruple $Y$-number $(0,0 T, 0 I, 0 F)$ is denoted by 0 , that is,

$$
\tilde{0}=(0,0 T, 0 I, 0 F) .
$$

If $Y$ has an order relation " $\leq$ ", then we define an order relation " $\ll$ " and the equality "=" on $\mathcal{N}_{q}(Y)$ as follows:

$$
\begin{aligned}
& \tilde{a} \ll \tilde{b} \Leftrightarrow a_{i} \leq b_{i} \text { for } i=1,2,3,4, \\
& \tilde{a}=\tilde{b} \Leftrightarrow a_{i}=b_{i} \text { for } i=1,2,3,4
\end{aligned}
$$

for all $\tilde{a}, \tilde{b} \in \mathcal{N}_{q}(Y)$. It is easy to verify that, if " $\leq$ " is a partial order on $Y$, then " $\ll$ " is a partial order on $\mathcal{N}_{q}(Y)$.

Definition 2 ([18]). Given a set $Y$ with a binary operation $*$ and a special number 0 , the neutrosophic quadruple $Y$-set $\mathcal{N}_{q}(Y)$ is called a neutrosophic quadruple BCK / BCI-algebra if $\left(\mathcal{N}_{q}(Y)\right.$; $\left.\tilde{*}, \tilde{0}\right)$ is a BCK / BCI-algebra.

\section{Implicative Neutrosophic Quadruple Ideals}

In this section, we first consider conditions for a the neutrosophic quadruple $Y$-set $\mathcal{N}_{q}(Y)$ to be a neutrosophic quadruple $B C I$-algebra. We define the notion of (commutative, implicative) neutrosophic quadruple $B C K$-algebra and investigate related properties.

Theorem 1. Given a set $Y$ with a binary operation $*$ and a special number 0 , if the neutrosophic quadruple $Y$-set $\mathcal{N}_{q}(Y)$ has a binary operation " $\tilde{*}$ " and a partial ordering " $\ll$ " such that

(1) $\quad(\tilde{x} \tilde{y} \tilde{y}) \tilde{*}(\tilde{x} \tilde{*} \tilde{z}) \ll \tilde{z} \tilde{*} \tilde{y}$,

(2) $\tilde{x} \tilde{*}(\tilde{x} \tilde{*} \tilde{y}) \ll \tilde{y}$,

(3) $\tilde{x} \tilde{*} \tilde{y}=\tilde{0} \Leftrightarrow \tilde{x} \ll \tilde{y}$ 
for all $\tilde{x}, \tilde{y}, \tilde{z} \in \mathcal{N}_{q}(Y)$, then $\mathcal{N}_{q}(Y)$ is a neutrosophic quadruple BCI-algebra.

Proof. Let $\tilde{x}, \tilde{y}, \tilde{z} \in \mathcal{N}_{q}(Y)$. Using conditions (1) and (3) of this theorem, we have

$$
((\tilde{x} \tilde{*} \tilde{y}) \tilde{*}(\tilde{x} \tilde{*} \tilde{z})) \tilde{*}(\tilde{z} \tilde{*} \tilde{y})=\tilde{0} .
$$

Assume that $\tilde{x} \tilde{*} \tilde{y}=\tilde{0}$ and $\tilde{y} \tilde{*} \tilde{x}=\tilde{0}$. Then, $\tilde{x} \ll \tilde{y}$ and $\tilde{y} \ll \tilde{x}$ by (3), which implies that $\tilde{x}=\tilde{y}$ by the anti-symmetry of $\ll$. By the condition (3) of this theorem and the reflexivity of $\ll$, we get $\tilde{x} \tilde{*} \tilde{x}=\tilde{0}$. Using conditions (2) and (3) of this theorem, we have

$$
(\tilde{x} \tilde{*}(\tilde{x} \tilde{*} \tilde{y})) \tilde{*} \tilde{y}=\tilde{0} .
$$

Putting $\tilde{y}=\tilde{0}$ in (12) implies that

$$
(\tilde{x} \tilde{x}(\tilde{x} \tilde{*} \tilde{0})) \tilde{*} \tilde{0}=\tilde{0} .
$$

If we substitute $\tilde{x} \tilde{*} \tilde{0}$ and $\tilde{x}$ for $\tilde{y}$ and $\tilde{z}$, respectively, in (11), then

$$
\begin{aligned}
\tilde{0} & =((\tilde{x} \tilde{*}(\tilde{x} \tilde{0} \tilde{0})) \tilde{*}(\tilde{x} \tilde{*} \tilde{x})) \tilde{*}(\tilde{x} \tilde{*}(\tilde{x} \tilde{*} \tilde{0})) \\
& =((\tilde{x} \tilde{*}(\tilde{x} \tilde{0} \tilde{0})) \tilde{*} \tilde{0}) \tilde{x}(\tilde{x} \tilde{x}(\tilde{x} \tilde{0} \tilde{0})) \\
& =\tilde{0} \tilde{*}(\tilde{x} \tilde{*}(\tilde{x} \tilde{0} \tilde{0})) .
\end{aligned}
$$

Hence, $\tilde{x} \tilde{*}(\tilde{x} \tilde{*} \tilde{0})=\tilde{0}$. On the other hand, we get $(\tilde{x} \tilde{*} \tilde{0}) \tilde{*} \tilde{x}=(\tilde{x} \tilde{*}(\tilde{x} \tilde{*} \tilde{x})) \tilde{*} \tilde{x}=\tilde{0}$. It follows that $\tilde{x} \tilde{*} \tilde{0}=\tilde{x}$. Hence, $\left(\mathcal{N}_{q}(Y) ; \tilde{*}, \tilde{0}\right)$ is a $B C I$-algebra, and therefore $\mathcal{N}_{q}(Y)$ is a neutrosophic quadruple $B C I$-algebra.

Definition 3. Given a set $Y$ with a binary operation $*$ and a special number 0 , the neutrosophic quadruple $Y$-set $\mathcal{N}_{q}(Y)$ is called a (commutative, implicative) neutrosophic quadruple $B C K$-algebra if $\left(\mathcal{N}_{q}(Y) ; \tilde{*}, \tilde{0}\right)$ is a (commutative, implicative) BCK-algebra.

Example 1. Given a set $Y=\{0, a\}$, consider the neutrosophic quadruple $Y$-set as follows:

$$
\mathcal{N}_{q}(Y)=\left\{\tilde{\alpha}_{0}, \tilde{\alpha}_{1}, \tilde{\alpha}_{2}, \tilde{\alpha}_{3}, \tilde{\alpha}_{4}, \tilde{\alpha}_{5}, \tilde{\alpha}_{6}, \tilde{\alpha}_{7}, \tilde{\alpha}_{8}, \tilde{\alpha}_{9}, \tilde{\alpha}_{10}, \tilde{\alpha}_{11}, \tilde{\alpha}_{12}, \tilde{\alpha}_{13}, \tilde{\alpha}_{14}, \tilde{\alpha}_{15}\right\},
$$

where

$$
\begin{gathered}
\tilde{\alpha}_{0}=(0,0 T, 0 I, 0 F), \tilde{\alpha}_{1}=(0,0 T, 0 I, a F), \tilde{\alpha}_{2}=(0,0 T, a I, 0 F), \tilde{\alpha}_{3}=(0,0 T, a I, a F), \\
\tilde{\alpha}_{4}=(0, a T, 0 I, 0 F), \tilde{\alpha}_{5}=(0, a T, 0 I, a F), \tilde{\alpha}_{6}=(0, a T, a I, 0 F), \tilde{\alpha}_{7}=(0, a T, a I, a F), \\
\tilde{\alpha}_{8}=(a, 0 T, 0 I, 0 F), \tilde{\alpha}_{9}=(a, 0 T, 0 I, a F), \tilde{\alpha}_{10}=(a, 0 T, a I, 0 F), \tilde{\alpha}_{11}=(a, 0 T, a I, a F), \\
\tilde{\alpha}_{12}=(a, a T, 0 I, 0 F), \tilde{\alpha}_{13}=(a, a T, 0 I, a F), \tilde{\alpha}_{14}=(a, a T, a I, 0 F), \tilde{\alpha}_{15}=(a, a T, a I, a F) .
\end{gathered}
$$

Define a binary operation “ $\widetilde{*}$ ” on $\mathcal{N}_{q}(Y)$ by Table 1 . 
Table 1. Binary operation “ $\tilde{*} "$.

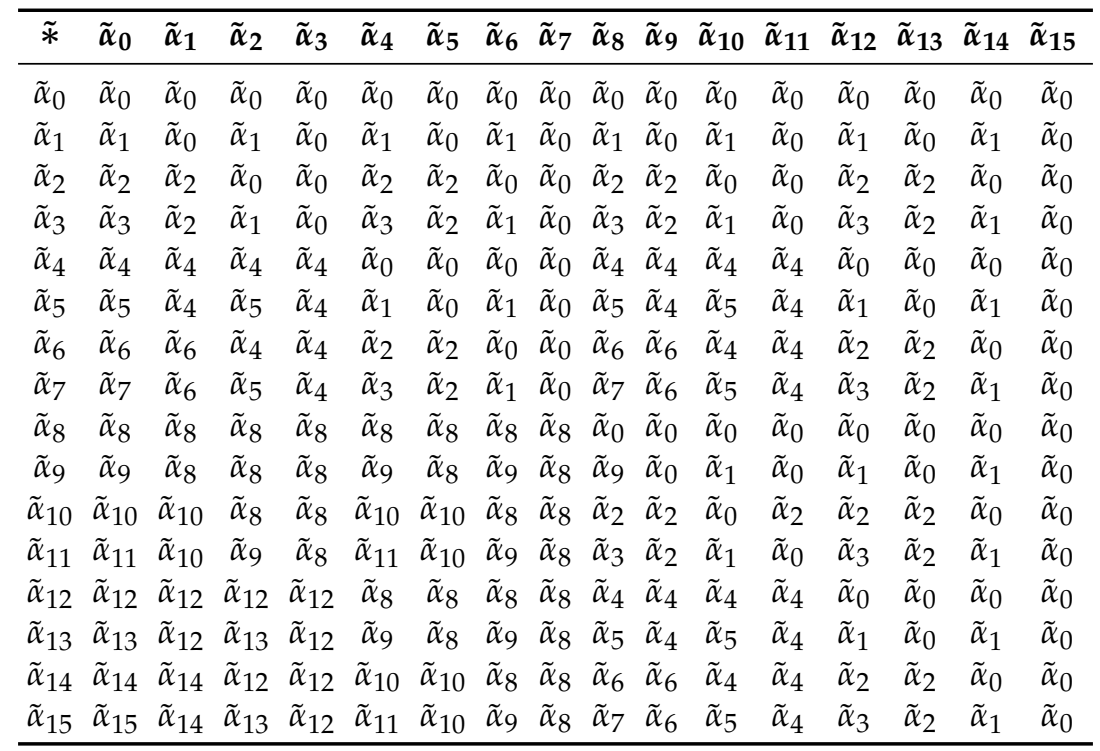

Then, $\mathcal{N}_{q}(Y)$ is a (commutative, implicative) neutrosophic quadruple BCK-algebra.

Lemma 1 ([18]). If $Y$ is a $B C K / B C I$-algebra, then $\mathcal{N}_{q}(Y)$ is a neutrosophic quadruple BCK/BCI-algebra.

Theorem 2. If $Y$ is an implicative BCK-algebra, then the neutrosophic quadruple $Y$-set $\mathcal{N}_{q}(Y)$ is an implicative neutrosophic quadruple BCK-algebra.

Proof. Let $Y$ be an implicative $B C K$-algebra. Then, $Y$ is a $B C K$-algebra, and so $\mathcal{N}_{q}(Y)$ is a neutrosophic quadruple $B C K$-algebra by Lemma 1 . Let $\tilde{x}, \tilde{y} \in \mathcal{N}_{q}(Y)$. Then, $x_{i} *\left(y_{i} * x_{i}\right)=x_{i}$ for all $i=1,2,3,4$ since $x_{i}, y_{i} \in Y$ and $Y$ is an implicative $B C K$-algebra. Hence,

$$
\begin{aligned}
\tilde{x} \tilde{*}(\tilde{y} \tilde{*}) & =\left(x_{1} *\left(y_{1} * x_{1}\right),\left(x_{2} *\left(y_{2} * x_{2}\right)\right) T,\left(x_{3} *\left(y_{3} * x_{3}\right)\right) I,\left(x_{4} *\left(y_{4} * x_{4}\right)\right) F\right) \\
& =\left(x_{1}, x_{2} T, x_{3} I, x_{4} F\right)=\tilde{x}
\end{aligned}
$$

and therefore $\mathcal{N}_{q}(Y)$ is an implicative neutrosophic quadruple $B C K$-algebra.

Lemma 2 ([21]). If $Y$ is a commutative BCK-algebra, then the neutrosophic quadruple $Y$-set $\mathcal{N}_{q}(Y)$ is a commutative neutrosophic quadruple BCK-algebra.

Since every implicative $B C K$-algebra is a commutative $B C K$-algebra, we have the following corollary.

Corollary 1. Every neutrosophic quadruple $Y$-set $\mathcal{N}_{q}(Y)$ based on an implicative BCK-algebra $Y$ is a commutative neutrosophic quadruple BCK-algebra.

Proposition 1. The neutrosophic quadruple $Y$-set $\mathcal{N}_{q}(Y)$ based on an implicative BCK-algebra $Y$ satisfies the following assertions:

(1) $\quad(\tilde{x} \tilde{*}(\tilde{x} \tilde{*} \tilde{y})) \tilde{*}(\tilde{x} \tilde{*} \tilde{y})=\tilde{y} \tilde{*}(\tilde{y} \tilde{*} \tilde{x})$,

(2) $\quad(\tilde{x} \tilde{*}(\tilde{x} \tilde{*} \tilde{y})) \tilde{*}(\tilde{y} \tilde{*} \tilde{x})=\tilde{y} \tilde{*}(\tilde{y} \tilde{*} \tilde{x})$,

(3) $\quad(\tilde{x} \tilde{*}(\tilde{x} \tilde{*} \tilde{y})) \tilde{*}(\tilde{x} \tilde{*} \tilde{y})=(\tilde{y} \tilde{*}(\tilde{y} \tilde{*} \tilde{x})) \tilde{*}(\tilde{y} \tilde{*} \tilde{x})$

for all $\tilde{x}, \tilde{y} \in \mathcal{N}_{q}(Y)$. 
Proof. Let $Y$ be an implicative $B C K$-algebra. Then,

$$
\left(x_{i} *\left(x_{i} * y_{i}\right)\right) *\left(x_{i} * y_{i}\right)=y_{i} *\left(y_{i} * x_{i}\right)
$$

for all $x_{i}, y_{i} \in Y$ with $i=1,2,3,4$. Thus,

$$
\begin{aligned}
& \begin{aligned}
(\tilde{x} \tilde{*}(\tilde{x} \tilde{*} \tilde{y})) \tilde{*}(\tilde{x} \tilde{*} \tilde{y}) & =\left(\left(x_{1}, x_{2} T, x_{3} I, x_{4} F\right) \tilde{*}\left(\left(x_{1}, x_{2} T, x_{3} I, x_{4} F\right) \tilde{*}\left(y_{1}, y_{2} T, y_{3} I, y_{4} F\right)\right)\right) \\
& \tilde{*}\left(\left(x_{1}, x_{2} T, x_{3} I, x_{4} F\right) \tilde{*}\left(y_{1}, y_{2} T, y_{3} I, y_{4} F\right)\right) \\
= & \left(\left(x_{1} *\left(x_{1} * y_{1}\right)\right) *\left(x_{1} * y_{1}\right),\left(\left(x_{2} *\left(x_{2} * y_{2}\right)\right) *\left(x_{2} * y_{2}\right)\right) T,\right. \\
& \left.\left(\left(x_{3} *\left(x_{3} * y_{3}\right)\right) *\left(x_{3} * y_{3}\right)\right) I,\left(\left(x_{4} *\left(x_{4} * y_{4}\right)\right) *\left(x_{4} * y_{4}\right)\right) F\right)
\end{aligned} \\
& =\left(y_{1} *\left(y_{1} * x_{1}\right),\left(y_{2} *\left(y_{2} * x_{2}\right)\right) T,\left(y_{3} *\left(y_{3} * x_{3}\right)\right) I,\left(y_{4} *\left(y_{4} * x_{4}\right)\right) F\right) \\
& =\tilde{y} \tilde{*}(\tilde{y} \tilde{x}) .
\end{aligned}
$$

This proves (1). Similarly, we can prove (2) and (3).

Theorem 3. If the neutrosophic quadruple $Y$-set $\mathcal{N}_{q}(Y)$ based on a BCK-algebra $Y$ satisfies the condition (3) in Proposition 1, then it is an implicative neutrosophic quadruple BCK-algebra.

Proof. By Lemma 1, we know that $\mathcal{N}_{q}(Y)$ is a neutrosophic quadruple $B C K$-algebra. Let $\tilde{x}, \tilde{y} \in \mathcal{N}_{q}(Y)$. Then,

$$
\left(x_{i} *\left(x_{i} * y_{i}\right)\right) *\left(x_{i} * y_{i}\right)=\left(y_{i} *\left(y_{i} * x_{i}\right)\right) *\left(y_{i} * x_{i}\right)
$$

for all $i=1,2,3,4$. If we substitute $x_{i} * y_{i}$ for $y_{i}$ in (14), then

$$
\begin{aligned}
x_{i} * y_{i} & =\left(\left(x_{i} * y_{i}\right) * 0\right) * 0=\left(\left(x_{i} * y_{i}\right) *\left(\left(x_{i} * y_{i}\right) * x_{i}\right)\right) *\left(\left(x_{i} * y_{i}\right) * x_{i}\right) \\
& =\left(x_{i} *\left(x_{i} *\left(x_{i} * y_{i}\right)\right)\right) *\left(x_{i} *\left(x_{i} * y_{i}\right)\right) \\
& =\left(x_{i} * y_{i}\right) *\left(x_{i} *\left(x_{i} * y_{i}\right)\right) \\
& =\left(x_{i} *\left(x_{i} *\left(x_{i} * y_{i}\right)\right) * y_{i}\right. \\
& =\left(x_{i} * y_{i}\right) * y_{i} .
\end{aligned}
$$

It follows from (15) and (3) in Proposition 1 that

$$
x_{i} *\left(x_{i} * y_{i}\right)=\left(x_{i} *\left(x_{i} * y_{i}\right)\right) *\left(x_{i} * y_{i}\right)=\left(y_{i} *\left(y_{i} * x_{i}\right)\right) *\left(y_{i} * x_{i}\right)=y_{i} *\left(y_{i} * x_{i}\right) .
$$

Using (15) and (16), we have

$$
x_{i} *\left(x_{i} *\left(y_{i} * x_{i}\right)\right)=\left(y_{i} * x_{i}\right) *\left(\left(y_{i} * x_{i}\right) * x_{i}\right)=\left(\left(y_{i} * x_{i}\right) * x_{i}\right) *\left(\left(y_{i} * x_{i}\right) * x_{i}\right)=0 .
$$

Obviously, $\left(x_{i} *\left(y_{i} * x_{i}\right)\right) * x_{i}=0$. Hence, $x_{i} *\left(y_{i} * x_{i}\right)=x_{i}$, and thus

$$
\begin{aligned}
\tilde{x} \tilde{*}(\tilde{y} \tilde{*}) & =\left(x_{1} *\left(y_{1} * x_{1}\right),\left(x_{2} *\left(y_{2} * x_{2}\right)\right) T,\left(x_{3} *\left(y_{3} * x_{3}\right)\right) I,\left(x_{4} *\left(y_{4} * x_{4}\right)\right) F\right) \\
& =\left(x_{1}, x_{2} T, x_{3} I, x_{4} F\right)=\tilde{x} .
\end{aligned}
$$

Hence, $\left(\mathcal{N}_{q}(Y) ; \tilde{*}, \tilde{0}\right)$ is an implicative $B C K$-algebra, and therefore $\mathcal{N}_{q}(Y)$ is an implicative neutrosophic quadruple $B C K$-algebra.

Given subsets $I$ and $J$ of a $B C K$-algebra $Y$, consider the set

$$
\mathcal{N}_{q}(I, J):=\left\{(a, x T, y I, z F) \in \mathcal{N}_{q}(Y) \mid a, x \in I ; y, z \in J\right\},
$$


which is called the neutrosophic quadruple $(I, J)$-set. It is clear that the neutrosophic quadruple $(I, J)$-set is a subset of the neutrosophic quadruple $Y$-set $\mathcal{N}_{q}(Y)$.

Theorem 4. If I and J are implicative ideals of a BCK-algebra $Y$, then the neutrosophic quadruple $(I, J)$-set $\mathcal{N}_{q}(I, J)$ is an implicative ideal of the neutrosophic quadruple BCK-algebra $\mathcal{N}_{q}(Y)$.

Proof. Assume that $I$ and $J$ are implicative ideals of a $B C K$-algebra $Y$. Obviously, $0 \in \mathcal{N}_{q}(I, J)$. Let $\tilde{x}=\left(x_{1}, x_{2} T, x_{3} I, x_{4} F\right), \tilde{y}=\left(y_{1}, y_{2} T, y_{3} I, y_{4} F\right)$ and $\tilde{z}=\left(z_{1}, z_{2} T, z_{3} I, z_{4} F\right)$ be elements of $\mathcal{N}_{q}(Y)$ such that $(\tilde{x} \tilde{*}(\tilde{y} \tilde{x} \tilde{x})) \tilde{*} \tilde{z} \in \mathcal{N}_{q}(I, J)$ and $\tilde{z} \in \mathcal{N}_{q}(I, J)$. Then,

$$
\begin{aligned}
(\tilde{x} \tilde{*}(\tilde{y} \tilde{*} \tilde{x})) \tilde{*} \tilde{z}=( & \left(x_{1} *\left(y_{1} * x_{1}\right)\right) * z_{1},\left(\left(x_{2} *\left(y_{2} * x_{2}\right)\right) * z_{2}\right) T, \\
& \left.\left(\left(x_{3} *\left(y_{3} * x_{3}\right)\right) * z_{3}\right) I,\left(\left(x_{4} *\left(y_{4} * x_{4}\right)\right) * z_{4}\right) F\right) \in \mathcal{N}_{q}(I, J),
\end{aligned}
$$

and so $\left(x_{1} *\left(y_{1} * x_{1}\right)\right) * z_{1} \in I,\left(x_{2} *\left(y_{2} * x_{2}\right)\right) * z_{2} \in I,\left(x_{3} *\left(y_{3} * x_{3}\right)\right) * z_{3} \in J$ and $\left(x_{4} *\left(y_{4} * x_{4}\right)\right) * z_{4} \in$ $J$. Since $\tilde{z} \in \mathcal{N}_{q}(I, J)$, we have $z_{1}, z_{2} \in I$ and $z_{3}, z_{4} \in J$. Since $I$ and $I$ are implicative ideals of $Y$, it follows that $x_{1}, x_{2} \in I$ and $x_{3}, x_{4} \in J$. Hence, $\tilde{x}=\left(x_{1}, x_{2} T, x_{3} I, x_{4} F\right) \in \mathcal{N}_{q}(I, J)$, and therefore $\mathcal{N}_{q}(I, J)$ is an implicative ideal of $\mathcal{N}(Y)$.

Lemma 3 ([21]). If I and J are commutative ideals of a BCK-algebra $Y$, then the neutrosophic quadruple $(I, J)$-set $\mathcal{N}_{q}(I, J)$ is a commutative ideal of the neutrosophic quadruple BCK-algebra $\mathcal{N}_{q}(Y)$.

Since every implicative ideal is a commutative ideal, we have the following corollary.

Corollary 2. If I and J are implicative ideals of a BCK-algebra $Y$, then the neutrosophic quadruple $(I, J)$-set $\mathcal{N}_{q}(I, J)$ is a commutative ideal of the neutrosophic quadruple BCK-algebra $\mathcal{N}_{q}(Y)$.

The following example illustrates Theorem 4.

Example 2. Consider a BCK-algebra $Y=\{0, a, b, c\}$ in which the binary operation $*$ is given by Table 2,

Table 2. Binary operation " $*$ ".

\begin{tabular}{lllll}
\hline$*$ & $\mathbf{0}$ & $\boldsymbol{a}$ & $\boldsymbol{b}$ & $\boldsymbol{c}$ \\
\hline 0 & 0 & 0 & 0 & 0 \\
$a$ & $a$ & 0 & $a$ & 0 \\
$b$ & $b$ & $b$ & 0 & 0 \\
$c$ & $c$ & $b$ & $a$ & 0 \\
\hline
\end{tabular}

Then, the neutrosophic quadruple BCK-algebra $\mathcal{N}_{q}(Y)$ has 256 elements. Note that $I:=\{0, a\}$ and $J:=\{0, b\}$ are implicative ideals of $Y$. Hence, the neutrosophic quadruple $(I, J)$-set $\mathcal{N}_{q}(I, J)$ is given as follows:

$$
\mathcal{N}_{q}(I, J)=\{\tilde{0}, \tilde{1}, \tilde{2}, \tilde{3}, \tilde{4}, \tilde{5}, \tilde{6}, \tilde{7}, \tilde{8}, \tilde{9}, \tilde{10}, \tilde{11}, \tilde{12}, \tilde{13}, \tilde{14}, \tilde{15}\}
$$

and it is an implicative ideal of the neutrosophic quadruple $B C K$-algebra $\mathcal{N}_{q}(Y)$ where

$$
\begin{gathered}
\tilde{0}=(0,0 T, 0 I, 0 F), \tilde{1}=(0,0 T, 0 I, b F), \tilde{2}=(0,0 T, b I, 0 F), \tilde{3}=(0,0 T, b I, b F), \\
\tilde{4}=(0, a T, 0 I, 0 F), \tilde{5}=(0, a T, 0 I, b F), \tilde{6}=(0, a T, b I, 0 F), \tilde{7}=(0, a T, b I, b F), \\
\tilde{8}=(a, 0 T, 0 I, 0 F), \tilde{9}=(a, 0 T, 0 I, b F), \tilde{10}=(a, 0 T, b I, 0 F), \tilde{1}=(a, 0 T, b I, b F), \\
\tilde{12}=(a, a T, 0 I, 0 F), \tilde{13}=(a, a T, 0 I, b F), \tilde{14}=(a, a T, b I, 0 F), \tilde{15}=(a, a T, b I, b F) .
\end{gathered}
$$


Proposition 2. If I and J are implicative ideals of a BCK-algebra $Y$, then the neutrosophic quadruple $(I, J)$-set $\mathcal{N}_{q}(I, J)$ satisfies the following assertion:

$$
\left(\forall \tilde{x}, \tilde{y} \in \mathcal{N}_{q}(Y)\right)\left(\tilde{y} \tilde{*}(\tilde{y} \tilde{*} \tilde{x}) \in \mathcal{N}_{q}(I, J) \Rightarrow \tilde{x} \tilde{*}\left(\tilde{x} \tilde{y} \tilde{)} \in \mathcal{N}_{q}(I, J)\right) .\right.
$$

Proof. Assume that $I$ and $J$ are implicative ideals of a $B C K$-algebra $Y$ and $\tilde{y} \tilde{*}(\tilde{y} \tilde{*} \tilde{x}) \in \mathcal{N}_{q}(I, J)$ for all $\tilde{x}, \tilde{y} \in \mathcal{N}_{q}(Y)$. Then,

$$
\tilde{y} \tilde{*}(\tilde{y} \tilde{x})=\left(y_{1} *\left(y_{1} * x_{1}\right),\left(y_{2} *\left(y_{2} * x_{2}\right)\right) T,\left(y_{3} *\left(y_{3} * x_{3}\right)\right) I,\left(y_{4} *\left(y_{4} * x_{4}\right)\right) F\right) \in \mathcal{N}_{q}(I, J),
$$

and so $y_{1} *\left(y_{1} * x_{1}\right) \in I, y_{2} *\left(y_{2} * x_{2}\right) \in I, y_{3} *\left(y_{3} * x_{3}\right) \in J$ and $y_{4} *\left(y_{4} * x_{4}\right) \in J$. Since $x_{i} *\left(x_{i} * y_{i}\right) \leq$ $x_{i}$ for $i=1,2,3,4$, we have

$$
y_{i} * x_{i} \leq y_{i} *\left(x_{i} *\left(x_{i} * y_{i}\right)\right)
$$

which implies that

$$
\begin{aligned}
& \left(x_{i} *\left(x_{i} * y_{i}\right)\right) *\left(y_{i} *\left(x_{i} *\left(x_{i} * y_{i}\right)\right)\right) \leq\left(x_{i} *\left(x_{i} * y_{i}\right)\right) *\left(y_{i} * x_{i}\right) \\
& =\left(x_{i} *\left(y_{i} * x_{i}\right)\right) *\left(x_{i} * y_{i}\right) \leq y_{i} *\left(y_{i} * x_{i}\right)
\end{aligned}
$$

that is, $\left(\left(x_{i} *\left(x_{i} * y_{i}\right)\right) *\left(y_{i} *\left(x_{i} *\left(x_{i} * y_{i}\right)\right)\right)\right) *\left(y_{i} *\left(y_{i} * x_{i}\right)\right)=0 \in I \cap J$ for $i=1,2,3,4$. Since $y_{i} *$ $\left(y_{i} * x_{i}\right) \in I$ for $i=1,2, y_{j} *\left(y_{j} * x_{j}\right) \in J$ for $j=3,4$, and $I$ and $J$ are implicative ideals of $Y$, it follows from (10) that $x_{i} *\left(x_{i} * y_{i}\right) \in I$ for $i=1,2$, and $x_{j} *\left(x_{j} * y_{j}\right) \in J$ for $j=3,4$. Hence,

$$
\tilde{x} \tilde{*}(\tilde{x} \tilde{*} \tilde{y})=\left(x_{1} *\left(x_{1} * y_{1}\right),\left(x_{2} *\left(x_{2} * y_{2}\right)\right) T,\left(x_{3} *\left(x_{3} * y_{3}\right)\right) I,\left(x_{4} *\left(x_{4} * y_{4}\right)\right) F\right) \in \mathcal{N}_{q}(I, J) .
$$

This completes the proof.

Lemma 4 ([18]). If I and J are ideals of a BCK-algebra $Y$, then the neutrosophic quadruple $(I, J)$-set $\mathcal{N}_{q}(I, J)$ is an ideal of $\mathcal{N}_{q}(Y)$.

Theorem 5. Let I and J be ideals of a BCK-algebra Y such that

$$
\begin{aligned}
& (x * y) * y \in I(\text { resp., } J) \Rightarrow x * y \in I(\text { resp., } J), \\
& y *(y * x) \in I(\text { resp., } J) \Rightarrow x *(x * y) \in I(\text { resp., } J)
\end{aligned}
$$

for all $x, y \in Y$. Then, the neutrosophic quadruple $(I, J)$-set $\mathcal{N}_{q}(I, J)$ is an implicative ideal of $\mathcal{N}_{q}(Y)$.

Proof. If $I$ and $J$ are ideals of a $B C K$-algebra $Y$, then $\mathcal{N}_{q}(I, J)$ is an ideal of $\mathcal{N}_{q}(Y)$ by Lemma 4. Suppose $(\tilde{x} \tilde{*}(\tilde{y} \tilde{x} \tilde{x})) \tilde{z} \tilde{z} \in \mathcal{N}_{q}(I, J)$ and $\tilde{z} \in \mathcal{N}_{q}(I, J)$ for all $\tilde{x}, \tilde{y}, \tilde{z} \in \mathcal{N}_{q}(Y)$. Then,

$$
\begin{aligned}
&(\tilde{x} *(\tilde{y} * \tilde{x})) \tilde{*} z=(\left(x_{1} *\left(y_{1} * x_{1}\right)\right) * z_{1},\left(\left(x_{2} *\left(y_{2} * x_{2}\right)\right) * z_{2}\right) T, \\
&\left.\left(\left(x_{3} *\left(y_{3} * x_{3}\right)\right) * z_{3}\right) I,\left(\left(x_{4} *\left(y_{4} * x_{4}\right)\right) * z_{4}\right) F\right) \in \mathcal{N}_{q}(I, J)
\end{aligned}
$$

and $\tilde{z}=\left(z_{1}, z_{2} T, z_{3} I, z_{4} F\right) \in \mathcal{N}_{q}(I, J)$. It follows that $z_{1}, z_{2} \in I, z_{3}, z_{4} \in J,\left(x_{1} *\left(y_{1} * x_{1}\right)\right) * z_{1} \in I$, $\left(x_{2} *\left(y_{2} * x_{2}\right)\right) * z_{2} \in I,\left(x_{3} *\left(y_{3} * x_{3}\right)\right) * z_{3} \in J$ and $\left(x_{4} *\left(y_{4} * x_{4}\right)\right) * z_{4} \in J$. Since $I$ and $J$ are ideals of $Y$, we have $x_{1} *\left(y_{1} * x_{1}\right) \in I, x_{2} *\left(y_{2} * x_{2}\right) \in I, x_{3} *\left(y_{3} * x_{3}\right) \in J$ and $x_{4} *\left(y_{4} * x_{4}\right) \in J$. Since $\left(y_{i} *\left(y_{i} * x_{i}\right)\right) *\left(y_{i} * x_{i}\right) \leq x_{i} *\left(y_{i} * x_{i}\right)$ for $i=1,2,3,4$, it follows that $\left(y_{i} *\left(y_{i} * x_{i}\right)\right) *\left(y_{i} * x_{i}\right) \in I$ for $i=1,2$, and $\left(y_{j} *\left(y_{j} * x_{j}\right)\right) *\left(y_{j} * x_{j}\right) \in J$ for $j=3$, 4. Using (18), we obtain $y_{i} *\left(y_{i} * x_{i}\right) \in I$ for $i=1,2$, and $y_{j} *\left(y_{j} * x_{j}\right) \in J$ for $j=3,4$. It follows from (19) that

$$
x_{i} *\left(x_{i} * y_{i}\right) \in I \text { for } i=1,2 \text {, and } x_{j} *\left(x_{j} * y_{j}\right) \in J \text { for } j=3,4 .
$$


Note that $\left(x_{i} * y_{i}\right) * z_{i} \leq x_{i} * y_{i} \leq x_{i} *\left(y_{i} * x_{i}\right)$ for $i=1,2,3,4$. Thus, $\left(x_{i} * y_{i}\right) * z_{i} \in I$ for $i=1,2$, and $\left(x_{i} * y_{i}\right) * z_{i} \in J$ for $j=3,4$. Since $z_{1}, z_{2} \in I$ and $z_{3}, z_{4} \in J$, we obtain $x_{i} * y_{i} \in I$ for $i=1,2$, and $x_{j} * y_{j} \in J$ for $j=3,4$, which imply from (20) that $x_{1}, x_{2} \in I$ and $x_{3}, x_{4} \in J$. Hence, $\tilde{x}=\left(x_{1}, x_{2} T, x_{3} I, x_{4} F\right) \in \mathcal{N}_{q}(I, J)$, and therefore $\mathcal{N}_{q}(I, J)$ is an implicative ideal of $\mathcal{N}_{q}(Y)$.

Theorem 6. Let I and J be ideals of a BCK-algebra Y such that

$$
x *(y * x) \in I \text { (resp., } J) \Rightarrow x \in I \text { (resp., } J)
$$

for all $x, y \in Y$. Then, the neutrosophic quadruple $(I, J)$-set $\mathcal{N}_{q}(I, J)$ is an implicative ideal of $\mathcal{N}_{q}(Y)$.

Proof. If $I$ and $J$ are ideals of a $B C K$-algebra $Y$, then $\mathcal{N}_{q}(I, J)$ is an ideal of $\mathcal{N}_{q}(Y)$ by Lemma 4 . Let $\tilde{x}, \tilde{y}, \tilde{z} \in \mathcal{N}_{q}(Y)$ be such that $(\tilde{x} \tilde{*}(\tilde{y} \tilde{*} \tilde{x})) \tilde{*} \tilde{z} \in \mathcal{N}_{q}(I, J)$ and $\tilde{z} \in \mathcal{N}_{q}(I, J)$. Then,

$$
\begin{aligned}
(\tilde{x} \tilde{*}(\tilde{y} \tilde{*} \tilde{x})) \tilde{*} z=( & \left(x_{1} *\left(y_{1} * x_{1}\right)\right) * z_{1},\left(\left(x_{2} *\left(y_{2} * x_{2}\right)\right) * z_{2}\right) T, \\
& \left.\left(\left(x_{3} *\left(y_{3} * x_{3}\right)\right) * z_{3}\right) I,\left(\left(x_{4} *\left(y_{4} * x_{4}\right)\right) * z_{4}\right) F\right) \in \mathcal{N}_{q}(I, J)
\end{aligned}
$$

and $\tilde{z}=\left(z_{1}, z_{2} T, z_{3} I, z_{F}\right) \in \mathcal{N}_{q}(I, J)$. It follows that $z_{i} \in I,\left(x_{i} *\left(y_{i} * x_{i}\right)\right) * z_{i} \in I$ for $i=1,2$ and $z_{j} \in J,\left(x_{j} *\left(y_{j} * x_{j}\right)\right) * z_{j} \in J$ for $j=3,4$. Since $I$ and $J$ are ideals of $Y$, we have $x_{i} *\left(y_{i} * x_{i}\right) \in I$ for $i=1,2$ and $x_{j} *\left(y_{j} * x_{j}\right) \in J$ for $j=3,4$. Using (21), we get $x_{1}, x_{2} \in I$ and $x_{3}, x_{4} \in J$. Hence $\tilde{x}=$ $\left(x_{1}, x_{2} T, x_{3} I, x_{4} F\right) \in \mathcal{N}_{q}(I, J)$, and therefore $\mathcal{N}_{q}(I, J)$ is an implicative ideal of $\mathcal{N}_{q}(Y)$.

Lemma 5 ([20]). If I is an implicative ideal of a BCK-algebra $Y$, then every ideal A containing I is implicative.

Theorem 7. Let $A, B, I$ and $J$ be ideals of a $B C K$-algebra $Y$ such that $A \subseteq I$ and $B \subseteq J$. If $A$ and $B$ are implicative ideals of $Y$, then the neutrosophic quadruple $(I, J)$-set $\mathcal{N}_{q}(I, J)$ is an implicative ideal of $\mathcal{N}_{q}(Y)$.

Proof. If $A, B, I$ and $J$ are ideals of $Y$, then $\mathcal{N}_{q}(A, B)$ and $\mathcal{N}_{q}(I, J)$ are ideals of $\mathcal{N}_{q}(Y)$ by Lemma 4 and $\mathcal{N}_{q}(A, B) \subseteq \mathcal{N}_{q}(I, J)$. Since $A$ and $B$ are implicative ideals of $Y$, it follows from Theorem 4 that $\mathcal{N}_{q}(A, B)$ is an implicative ideal of $\mathcal{N}_{q}(Y)$. Therefore, the neutrosophic quadruple $(I, J)$-set $\mathcal{N}_{q}(I, J)$ is an implicative ideal of $\mathcal{N}_{q}(Y)$ by Lemma 5 .

\section{Conclusions}

Based on the concept of neutrosophic quadruple numbers which is introduced by Florentin Smarandache, Jun et al. have introduced the notion of neutrosophic quadruple $B C K / B C I$-numbers, and have studied neutrosophic quadruple $B C K / B C I$-algebras. As a continuation of Jun et al.'s paper which has been published in Axioms, we have introduced the notion of implicative neutrosophic quadruple $B C K$-algebras and have investigated several properties. Given a set $Y$, we have provided conditions for the neutrosophic quadruple $Y$-set $\mathcal{N}_{q}(Y)$ to be a neutrosophic quadruple $B C I$-algebra, and have considered conditions for the neutrosophic quadruple $Y$-set $\mathcal{N}_{q}(Y)$ to be an implicative neutrosophic quadruple $B C K$-algebra. Given subsets $I$ and $J$ of a $B C K$-algebra $Y$, we have discussed conditions for the neutrosophic quadruple $(I, J)$-set $\mathcal{N}_{q}(I, J)$ to be an implicative ideal of the neutrosophic quadruple $B C K$-algebra $\mathcal{N}_{q}(Y)$. In the forthcoming research and papers, we will continue these ideas and will define new notions. We will study several kinds of neutrosophic quadruple ideals in neutrosophic quadruple $B C K / B C I$-algebras.

Author Contributions: Conceiving the idea, G.M. and Y.B.J.; literature review, A.N.A., E.H.R.; writing-original draft preparation, G.M.; review and editing, G.M., A.N.A., E.H.R.; supervision, Y.B.J.

Funding: The first author is partially supported by the research grant S-0064-1439, Deanship of Scientific Research, University of Tabuk, Tabuk-71491, Saudi Arabia.

Acknowledgments: The authors are grateful to the learned referees for careful detailed reading and helpful comments/suggestions that improved the overall presentation of this paper. 
Conflicts of Interest: The authors declare no conflict of interest.

\section{References}

1. Smarandache, F. Neutrosophy, Neutrosophic Probability, Set, and Logic; ProQuest Information \& Learning: Ann Arbor, MI, USA, 1998; 105p. Available online: http:/ / fs.gallup.unm.edu/eBook-neutrosophics6.pdf (accessed on 28 June 2017).

2. Smarandache, F. A Unifying Field in Logics: Neutrosophic Logic. Neutrosophy, Neutrosophic Set, Neutrosophic Probability; American Reserch Press: Rehoboth, NM, USA, 1999.

3. Smarandache, F. Neutrosophic set-a generalization of the intuitionistic fuzzy set. Int. J. Pure Appl. Math. 2005, 24, 287-297.

4. Garg, H. Non-linear programming method for multi-criteria decision making problems under interval neutrosophic set environment. Appl. Intell. 2018, 48, 2199-2213. [CrossRef]

5. Garg, H. New logarithmic operational laws and their applications to multiattribute decision making for single-valued neutrosophic numbers. Cogn. Syst. Res. 2018, 52, 931-946. [CrossRef]

6. Muhiuddin, G.; Kim, S.J.; Jun, Y.B. Implicative N-ideals of BCK-algebras based on neutrosophic N-structures, Discrete Mathematics. Algorithms Appl. 2019, in press.

7. Muhiuddin, G.; Bordbar, H.; Smarandache, F.; Jun, Y.B. Further results on $(\in, \in)$-neutrosophic subalgebras and ideals in BCK/BCI-algebras. Neutrosophic Sets Syst. 2018, 20, 36-43.

8. Saeid, A.B.; Jun, Y.B. Neutrosophic subalgebras of $B C K / B C I$-algebras based on neutrosophic points. Ann. Fuzzy Math. Inform. 2017, 14, 87-97.

9. Jun, Y.B. Neutrosophic subalgebras of several types in BCK/BCI-algebras. Ann. Fuzzy Math. Inform. 2017, $14,75-86$.

10. Jun, Y.B.; Kim, S.J.; Smarandache, F. Interval neutrosophic sets with applications in BCK/BCI-algebra. Axioms 2018, 7, 23. [CrossRef]

11. Jun, Y.B.; Smarandache, F.; Bordbar, H. Neutrosophic $\mathcal{N}$-structures applied to $B C K / B C I$-algebras. Information 2017, 8, 128. [CrossRef]

12. Jun, Y.B.; Smarandache, F.; Song, S.Z.; Khan, M. Neutrosophic positive implicative $\mathcal{N}$-ideals in $B C K / B C I$-algebras. Axioms 2018, 7, 3. [CrossRef]

13. Khan, M.; Anis, S.; Smarandache, F.; Jun, Y.B. Neutrosophic $\mathcal{N}$-structures and their applications in Semigroups. Ann. Fuzzy Math. Inform. 2017, 14, 583-598.

14. Öztürk, M.A.; Jun, Y.B. Neutrosophic ideals in BCK/BCI-algebras based on neutrosophic points. J. Int. Math. Virtual Inst. 2018, 8, 1-17.

15. Song, S.Z.; Smarandache, F.; Jun, Y.B. Neutrosophic commutative $\mathcal{N}$-ideals in $B C K$-algebras. Information 2017, 8, 130. [CrossRef]

16. Smarandache, F. Neutrosophic quadruple numbers, refined neutrosophic quadruple numbers, absorbance law, and the multiplication of neutrosophic quadruple numbers. Neutrosophic Sets Syst. 2015, 10, 96-98.

17. Akinleye, S.A.; Smarandache, F.; Agboola, A.A.A. On neutrosophic quadruple algebraic structures. Neutrosophic Sets Syst. 2016, 12, 122-126.

18. Jun, Y.B.; Song, S.Z.; Smarandache, F.; Bordbar, H. Neutrosophic quadruple BCK/BCI-algebras. Axioms 2018, 7, 41. [CrossRef]

19. Huang, Y. BCI-Algebra; Science Press: Beijing, China, 2006.

20. Meng, J.; Jun, Y.B. BCK-Algebras; Kyungmoonsa Co.: Seoul, Korea, 1994.

21. Borzooei, R.A.; Takallo, M.M.; Jun, Y.B. Commutative neutrosophic quadruple ideals of neutrosophic quadruple BCK-algebras. Lobachevskii J. Math. 2019, submitted.

(C) 2019 by the authors. Licensee MDPI, Basel, Switzerland. This article is an open access article distributed under the terms and conditions of the Creative Commons Attribution (CC BY) license (http:/ / creativecommons.org/licenses/by/4.0/). 